\title{
Exploration of Coulomb explosion dynamics through excited vibrational states of molecules
}

\author{
Zhongyuan Zhou* and Shih-I Chu ${ }^{\dagger}$ \\ Department of Chemistry, University of Kansas, Lawrence, Kansas 66045, USA \\ (Received 30 August 2004; revised manuscript received 5 October 2004; published 31 January 2005)
}

\begin{abstract}
The fragmentation dynamics of $\mathrm{H}_{2}^{+}$molecular ions in intense laser fields is investigated by means of a high-precision $a b$ initio method beyond the Born-Oppenheimer approximation. Special attention is paid to the detailed Coulomb explosion (CE) mechanisms and dynamics through excited vibrational states of $\mathrm{H}_{2}^{+}$. A kinetic-energy release (KER) spectrum and CE phenomenon is predicted, in which the kinetic-energy distribution of $\mathrm{H}^{+}$ions exhibits a series of peaks separated by one photon energy. A proposed scheme for observation of the KER spectrum and CE dynamics is presented.
\end{abstract}

DOI: 10.1103/PhysRevA.71.011402

PACS number(s): 42.50.Hz, 33.80.Rv, 34.50.Gb

The study of ionization and molecular fragmentation is a subject of considerable interest in strong-field molecular physics. In particular, the responses of the simplest prototype molecular systems, $\mathrm{H}_{2}^{+}\left(\mathrm{D}_{2}^{+}\right)$, in intense laser fields have been extensively studied in the recent past [1-10]. Figure 1(a) displays some of the major fragmentation mechanisms arising from above-threshold dissociation (ATD) [11], dissociation via chemical bond softening (BSD) [12] and bond hardening $[13,14]$, Coulomb explosion (CE) by chargeresonance-enhanced ionization (CREI) [15], and CE by recollision of electrons $[2,3,16]$, etc. These mechanisms produce distinct peaks in the kinetic-energy release (KER) spectrum of $\mathrm{H}^{+}$that have been observed experimentally. The KER spectrum reflects a nuclear state at the time when $\mathrm{H}_{2}{ }^{+}$ breaks up [17].

However, most of the KER spectra of $\mathrm{H}^{+}$ions obtained to date are not the spectra of the "pure" $\mathrm{H}_{2}{ }^{+}$fragmentation because the experiments were performed using $\mathrm{H}_{2}$ molecules as the initial primary target $[8,9]$. In these experiments, the $\mathrm{H}_{2}{ }^{+}$ is formed from $\mathrm{H}_{2}$ in a very fast ionization process at the leading edge of the laser pulse, the ionization of $\mathrm{H}_{2}$ does not affect the fragmentation process of $\mathrm{H}_{2}{ }^{+}$, and the KER spectrum of $\mathrm{H}^{+}$is only produced by dissociation and $\mathrm{CE}$ of $\mathrm{H}_{2}{ }^{+}$. Nevertheless, in almost all the experiments with $\mathrm{H}_{2}$, only parts of the $\mathrm{H}_{2}$ molecules are ionized to $\mathrm{H}_{2}{ }^{+}$ions [8] and the ejected electron in the ionization process is highly correlated with its parent $\mathrm{H}_{2}{ }^{+}$ion $[1,10]$. Moreover, recent theoretical studies [16] indicate that the correlation effect between the electrons and ions plays an important role in the fragmentation dynamics of $\mathrm{D}_{2}$ and have strong influence on the subsequent dissociation. In particular, it has been shown in recent experiments using $\mathrm{H}_{2}{ }^{+}$ions $[8,9]$ that the KER spectra of $\mathrm{H}^{+}$ deviate greatly from those obtained from the experiments with $\mathrm{H}_{2}$. Thus, the fragmentation dynamics of $\mathrm{H}_{2}{ }^{+}$obtained indirectly from the $\mathrm{H}_{2}$ experiments is not consistent with that obtained by the direct $\mathrm{H}_{2}^{+}$experiments $[1,9]$.

In intense laser fields, the electron of $\mathrm{H}_{2}{ }^{+}$can be excited and ionized. If the electron is ionized, the $\mathrm{H}_{2}{ }^{+}$ion breaks up via direct Coulomb explosion (DCE), producing $\mathrm{H}^{+}$ions. If

\footnotetext{
*Electronic address: zyzhou@ku.edu

†Electronic address: sichu@ku.edu
}

the electron is excited to continuum vibrational states, the $\mathrm{H}_{2}{ }^{+}$can dissociate via these states directly or be further ionized by the laser [see Fig. 1(a)]. The latter process, which will be called excitation-ionization-dissociation (EID) hereafter, is a two-step process, in which the ionization of $\mathrm{H}_{2}{ }^{+}$in intermediate continuum vibrational states induces $\mathrm{CE}$. The experiments of ion-induced molecular fragments $[18,19]$ show that the excited states of the transient molecular ions have strong influence on KER spectra. Due to this influence, the KER spectra have fine structures that differ greatly from the DCE spectra. This demonstrates the limitation of the conventional DCE picture to explain molecular fragmentation dynamics. In addition, recent experiments of laser-induced fragmentation of $\mathrm{H}_{2}$ [1] show that the tunneling ionization of $\mathrm{H}_{2}$ does not follow the Franck-Condon principle due to the rapid variation of the ionization rate with internuclear separation. Thus the interpretations of earlier experiments may have to be reconsidered [1].

In this paper, we report on a KER spectrum and CE dy-
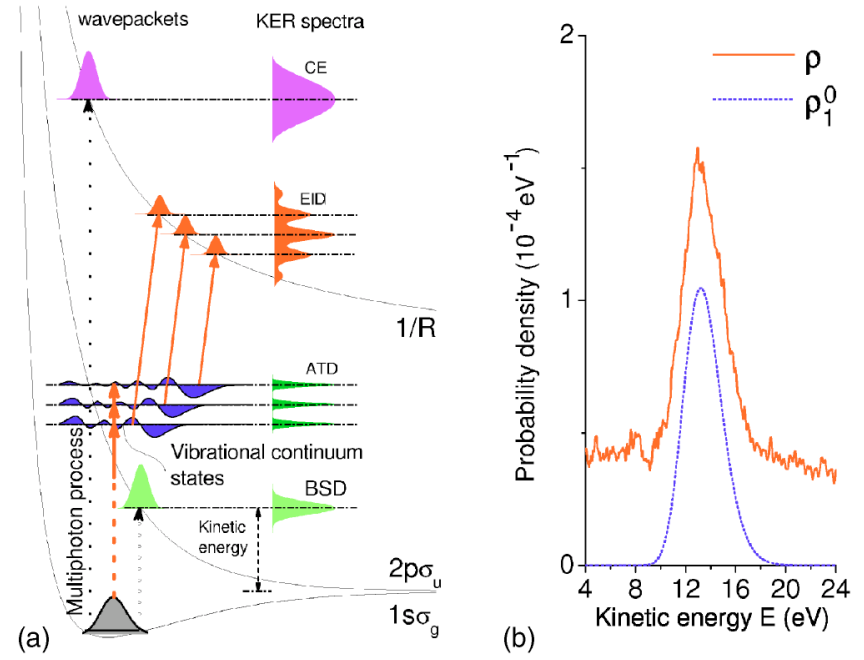

FIG. 1. Dissociative processes and the KER spectrum of $\mathrm{H}_{2}{ }^{+}$in intense laser fields. (a) A sketch of the various dissociative processes. (b) The CE-KER spectrum of $\mathrm{H}^{+}$for $\mathrm{H}_{2}{ }^{+}$with an initial state of the ground EV eigenstate $(1,0)$. The laser field is a $\sin ^{2}$-shaped pulse with $I_{0}=1 \times 10^{15} \mathrm{~W} / \mathrm{cm}^{2}, \lambda=790 \mathrm{~nm}$, and $T=90$ o.c. $\rho$ is the total KER spectra and $\rho_{1}^{0}$ is the DCE-KER spectra. 
namics. We assume that the $\mathrm{H}_{2}{ }^{+}$ions are well prepared in a given initial vibrational state $[8,9]$ and study the dynamics of intense laser- $\mathrm{H}_{2}{ }^{+}$interaction by means of a high-precision $a b$ initio method beyond the Born-Oppenheimer approximation (BBO). Special attention is paid to the correlation between electron and ion, as well as the KER spectrum of $\mathrm{H}^{+}$produced in the EID process. A kind of CE-KER spectrum of $\mathrm{H}^{+}$ with the fine structure $[7,10]$ associated with the EID of continuum vibrational states of $\mathrm{H}_{2}{ }^{+}$is predicted. This spectrum consists of a sequence of peaks separated by one photon energy and can be observed through initially prepared, highly excited vibrational states of $\mathrm{H}_{2}^{+}$using the techniques already available in the experiments $[8,9]$.

Suppose that the laser pulse is much shorter than the typical rotational period of a molecule and the molecular axis is aligned parallell to the linearly polarized laser field. In this case, the rotational degree of freedom can be frozen and the internal motions of the three-body $\mathrm{H}_{2}{ }^{+}$system can be described mathematically in a three-dimensional (3D) coordinate system of the center of mass: two prolate spheroidal coordinates $(\mu, \nu)(0 \leqslant \mu<\infty, 0 \leqslant \nu \leqslant \pi, 0 \leqslant \phi \leqslant 2 \pi$, where $\phi$ is fixed) for electronic motion, and an internuclear separation $R(0 \leqslant R \leqslant \infty)$ for nuclear vibration [20]. For each internuclear separation $R$, the electronic eigenenergy $E_{n}(R)$ (the potential energy for nuclear vibration) and eigenfunction $\psi_{n}(\mu, \nu, R)$ are calculated accurately and efficiently by solving the eigenvalue equation of a field-free fixed-nuclei electronic Hamiltonian in terms of a two-center generalized pseudospectral (GPS) method [21]. The GPS method allows nonuniform and optimal spatial grid discretization. Highprecision electronic eigenenergy and eigenfunction can be obtained with the use of only a modest number of grid points [21]. In order to take good care of the correlation between electron and ion, we expand the total time-dependent wave function $\Psi(\mu, \nu, R, t)$ in the electronic eigenfunctions $\Psi(\mu, \nu, R, t)=\Sigma_{n} \varphi_{n}(R, t) \psi_{n}(\mu, \nu, R)$ in the BBO framework. The time-dependent nuclear wave function of the $n$th electronic state, $\varphi_{n}(R, t)$, is calculated from the equation of motion (in a.u.)

$$
\begin{aligned}
i \frac{\partial}{\partial t} \varphi_{n}(R, t)= & {\left[-\frac{1}{2 m} \frac{\partial^{2}}{\partial R^{2}}+E_{n}(R)\right] \varphi_{n}(R, t)+\sum_{n^{\prime}}\left[V_{n n^{\prime}}(R, t)\right.} \\
& \left.+\zeta_{n n^{\prime}}(R) \frac{\partial}{\partial R}+\xi_{n n^{\prime}}(R)\right] \varphi_{n^{\prime}}(R, t)
\end{aligned}
$$

where $m$ is the reduced mass of nuclei, $V_{n n^{\prime}}(R, t)$ $=\left.\left\langle\psi_{n}|V(\mu, \nu, R, t)| \psi_{n^{\prime}}\right\rangle\right|_{R}$, (" $\left.\right|_{R}$ " means that the integral is performed for the fixed $R), \quad V(\mu, \nu, R, t)$ $=(D / 2) \varepsilon(t) R \cosh \mu \cos \nu$ is the interaction potential of $\mathrm{H}_{2}{ }^{+}$ with the laser field $\varepsilon(t)$ in the length gauge and electric dipole approximation, $D=1+1 / M$, and $M$ is the total mass of $\mathrm{H}_{2}{ }^{+} \cdot \zeta_{n n^{\prime}}(R)=-\left.(1 / m)\left\langle\psi_{n}|\partial / \partial R| \psi_{n^{\prime}}\right\rangle\right|_{R}$ and $\xi_{n n^{\prime}}(R)=-(1 / 2 m)$ $\times\left.\left\langle\psi_{n}\left|\partial^{2} / \partial R^{2}\right| \psi_{n^{\prime}}\right\rangle\right|_{R}$ are correction terms to the BornOppenheimer approximation (BO) since Eq. (1) reduces to an equation of the $\mathrm{BO}$ if $\zeta_{n n^{\prime}}(R)=\xi_{n n^{\prime}}(R)=0$. To solve Eq. (1), we use the Fourier-grid Hamiltonian (FGH) method [22] to discretize the $R$ coordinate and the second-order split- operator technique $[23,24]$ to propagate the time-dependent nuclear wave function.

Further, we expand the nuclear wave function $\varphi_{n}(R, t)$ in a complete set of nuclear vibrational eigenfunctions $\left\{\phi_{n v}(R)\right\}$ : $\varphi_{n}(R, t)=\Sigma_{v} \alpha_{n v}(t) \phi_{n v}(R)$, where the expansion coefficient $\alpha_{n v}(t)$ is the probability amplitude on an electronicvibrational $(\mathrm{EV})$ state $(n, v)$. The CE-KER spectrum of $\mathrm{H}^{+}$, which is described by the probability density of $\mathrm{H}^{+}$fragments, is calculated by $\int d E_{e}\left|\alpha_{E_{e} E}(t)\right|^{2}$, where $E_{e}$ is electronic energy in continuum electronic states, and $E$ refers to the sum energy shared by the fragments. This method is hard to use because $E_{e}$ depends on $R$ and it is impossible to find an exact $R$-independent $E_{e}$ for the integral.

An equivalent method is to project the total wave function to the eigenfunction of Hamiltonian with ionizationdissociation of $\mathrm{H}_{2}^{+}$. This Hamiltonian can be written as $H_{C}(\mathbf{r}, R)=H_{n}(\mathbf{r}, R)+H_{f e}(\mathbf{r}, R)$, where $\mathbf{r}$ is the electronic coordinate, $H_{n}=-\nabla_{R}^{2} / 2 m+1 / R$ is the Hamiltonian of nuclear motion, and $H_{f e}=-\nabla_{r}^{2} / 2$ is the Hamiltonian of a free electron. The eigenfunction of $H_{C}$ can be written as $\Phi_{C}(\mathbf{p}, E, \mathbf{r}, R)=\lambda(\mathbf{p}, \mathbf{r}) \chi(E, R)$, where $\chi(E, R)$ and $E(\geqslant 0)$ are the eigenfunction and the eigenenergy of $H_{n}$, and $\lambda(\mathbf{p}, \mathbf{r})$ and $p^{2} / 2$ ( $\mathbf{p}$ is the eigenmomentum of the free electron) are the eigenfunction and the eigenenergy of $H_{f e}$. The probability density of $\mathrm{H}^{+}$with kinetic energy $E$ is calculated by $\widetilde{\rho}(E)$ $=\int\left|w\left(\mathbf{p}, E, \tau_{f}\right)\right|^{2} d \mathbf{p}$, where the probability amplitude $w\left(\mathbf{p}, E, \tau_{f}\right)$ is obtained by projecting the time-dependent wave function $\Psi(\mu, \nu, R, t)$ at the end of the laser pulse $\tau_{f}$ to the $\mathrm{CE}$ eigenfunction $\Phi_{C}(\mathbf{p}, E, \mathbf{r}, R)$. For $\mathrm{H}_{2}{ }^{+}$, the interference between electronic states is very small [20] and can be neglected in the calculation of probability density. Furthermore, the electronic eigenfunction $\psi_{n}(\mathbf{r}, R)$ is a slowlyvarying function of $R$ [20]. In particular, $\psi_{n}(\mathbf{r}, R)$ does not change with $R$ under the BO. Thus to a good approximation, we assume that $\psi_{n}(\mathbf{r}, R)$ is independent of $R$ when calculating $w\left(\mathbf{p}, E, \tau_{f}\right)$. In this case, the probability density is calculated by $\widetilde{\rho}(E)=\Sigma_{n} \rho_{n}(E)$, where $\rho_{n}(E)$ is the contribution of $\mathrm{CE}$ from the $n$th electronic state given by $\rho_{n}(E)$ $=\left|\left\langle\chi(E, R) \mid \varphi_{n}\left(R, \tau_{f}\right)\right\rangle\right|^{2}$. If we ignore again the interference between vibrational eigenstates, the total probability density can be simplified to $\rho(E)=\Sigma_{n v} \rho_{n}^{v}(E)$, where $\rho_{n}^{v}(E)$ is the probability density from an $\mathrm{EV}$ state $(n, v)$ and is given by

$$
\rho_{n}^{v}(E)=\left|\alpha_{n v}\left(\tau_{f}\right)\left\langle\chi(E, R) \mid \phi_{n v}(R)\right\rangle\right|^{2} .
$$

We will illustrate that the main effect of the interference between vibrational states is to make the spectrum $\tilde{\rho}(E)$ exhibit some small oscillations. Because the sums to both $n$ and $v$ are over discrete and continuum states, the total KER spectrum contains all the contributions from various $\mathrm{CE}$ processes, including those from discrete EV states by direct removal of the electron. Equation (2) shows that the probability density from an EV state $(n, v), \rho_{n}^{v}$, is proportional to both the probability amplitude populated on this state $\alpha_{n v}$ and the ionization-dissociation probability amplitude from this state $\left\langle\chi \mid \phi_{n v}\right\rangle$. Suppose that the initial state of $\mathrm{H}_{2}^{+}$is an EV eigenstate $\left(n_{0}, v_{0}\right)$ and the DCE probability density from this state is $\rho_{n_{0}}^{v_{0}}(E)$, then $\rho^{*}(E)=\rho(E)-\rho_{n_{0}}^{v_{0}}(E)$ is the 


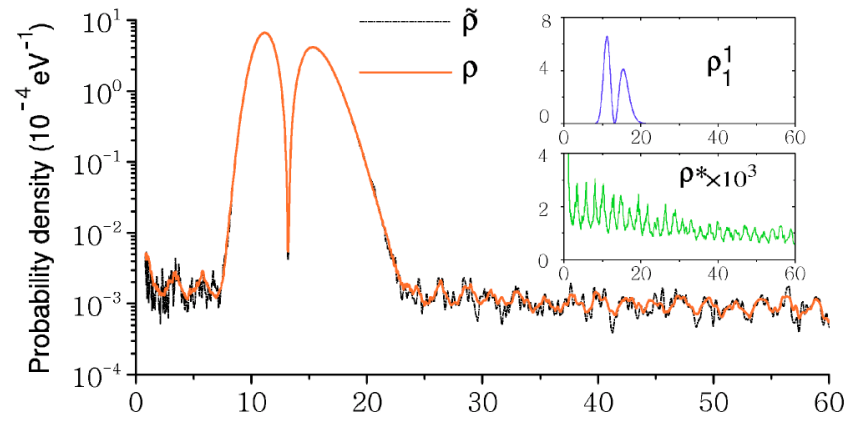

(a)

Kinetic energy $E(\mathrm{eV})$

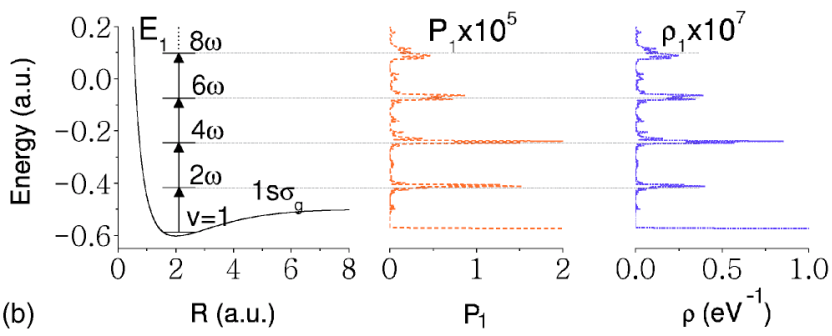

FIG. 2. $\mathrm{CE}$ of $\mathrm{H}_{2}{ }^{+}$with an excited initial EV state $(1,1)$. The laser field is a $\sin ^{2}$-shaped pulse with $I_{0}=5 \times 10^{13} \mathrm{~W} / \mathrm{cm}^{2}, \lambda$ $=532 \mathrm{~nm}$, and $T=128$ o.c. (a) The KER spectrum of $\mathrm{H}^{+} . \tilde{\rho}$ and $\rho$ are the total KER spectra with and without interference. $\rho_{1}^{1}$ in the upper inset, and $\rho^{*}$ in the lower inset, are the DCE spectra and EID spectra, respectively. (b) The potential energy $E_{1}(R)$ of $1 \mathrm{~s} \sigma_{g}$, population probability $P_{1}$ on $1 \mathrm{~s} \sigma_{g}$, and ionization-dissociation probability density $\rho_{1}$ from $1 \mathrm{~s} \sigma_{g}$.

probability density from all the other EV states. In the following, we will focus our investigation on the physical mechanism for producing $\rho^{*}(E)$.

We first applied the method to the investigation of the fragmentation of $\mathrm{H}_{2}{ }^{+}$initially prepared in the ground $\mathrm{EV}$ eigenstate $\left(n_{0}, v_{0}\right)=(1,0)$. The parameters of strong fs $\sin ^{2}$-shaped laser pulse are laser peak intensity $I_{0}=1$ $\times 10^{15} \mathrm{~W} / \mathrm{cm}^{2}$, wavelength $\lambda=790 \mathrm{~nm}$, and pulse duration $T=90$ optical cycles (o.c.) $(\approx 240 \mathrm{fs})$, which is an approximate pulse width with 80 fs full width at half maximum (FWHM) of a Gaussian pulse used in a recent experiment [7]. To achieve converged results, the boundary is set at $R_{\max }=35 \mathrm{a} . \mathrm{u}$. and up to 20 electronic states are included in the calculation. In Fig. 1(b), we show the total KER spectra $\rho$ and the DCE-KER spectra $\rho_{1}^{0}$. In the total spectra, there is a main peak located around $13.3 \mathrm{eV}$, extending from 10.5 to $17.1 \mathrm{eV}$. This peak is mainly produced from DCE of the initial state. It provides each $\mathrm{H}^{+}$with the kinetic energy up to $8.5 \mathrm{eV}$. This agrees well with the observation in the experiment [7]. However, Fig. 1(b) shows that the total spectra is quite different from the DCE spectra.

To get further insight into the fragmentation of $\mathrm{H}_{2}{ }^{+}$, we calculate the KER spectrum from the first excited vibrational state $\left(n_{0}, v_{0}\right)=(1,1)$ of $\mathrm{H}_{2}{ }^{+}$. The parameters of the $\sin ^{2}$-shaped laser pulse are $I_{0}=5 \times 10^{13} \mathrm{~W} / \mathrm{cm}^{2}, \lambda=532 \mathrm{~nm}$, and $T=128$ o.c. In Fig. 2(a) we display the total KER spectra with interference, $(\widetilde{\rho})$, and without interference $(\rho)$. The main difference between $\tilde{\rho}$ and $\rho$ is that $\widetilde{\rho}$ has some small oscillations around $\rho$. Thus, $\rho$ can be used to analyze the
KER spectra from different EV states without the loss of the main physical features. Figure 2(a) shows that the total spectra $\rho$ has two main peaks and a series of subpeaks. The two main peaks extending from 7.5 to $22.3 \mathrm{eV}$ are produced from the DCE of the initial state. They are much larger than those of the subpeaks. In order to explore the origin of the subpeaks, the DCE-KER spectra $\rho_{1}^{1}$ is separated from the total KER spectra $\rho$ and is plotted in the upper inset. The remain$\operatorname{der} \rho^{*}=\rho-\rho_{1}^{1}$ is displayed in the lower inset. The $\rho_{1}^{1}$ has two peaks located at 11.2 and $15.3 \mathrm{eV}$, while the $\rho^{*}$ has a sequence of peaks separated by one photon energy.

To probe the underlying mechanism responsible for the spectral feature of $\rho^{*}$, we calculate population probability on the EV state $(n, v), P_{n}(E)=\left|\alpha_{n v}(t)\right|^{2}$, and the ionizationdissociation probability density from the $n$th electronic state $\rho_{n}(E)$. For a continuum vibrational state $v$ of the $n$th electronic state, the kinetic energy in $P_{n}(E)$ is calculated by $E$ $=E_{n v}-E_{n D}$, where $E_{n v}$ is the eigenenergy of the EV continuum state $(n, v)$ and $E_{n D}$ is the dissociation energy of the $n$th electronic state. Because of the parity of the electron, if the initial state is a vibrational state of $1 s \sigma_{g}$, then only even number multiphoton processes are allowed for the transitions among the vibrational states of $1 s \sigma_{g}$ and only odd number multiphoton processes are possible for the transitions between the vibrational states of $1 s \sigma_{g}$ and those of $2 p \sigma_{u}$, etc. In Fig. 2(b) we plot the potential energy $E_{1}(R)$ of $1 s \sigma_{g}$, the population probability $P_{1}(E)$, and the ionization-dissociation probability density $\rho_{1}(E)$ at the end of the laser pulse. It is shown that $\rho_{1}$ is very similar to $P_{1}$, demonstrating that $\rho_{1}$ is proportional to $P_{1} . P_{1}$ is the probability on the highly excited and continuum vibrational states of $1 s \sigma_{g}$ [20], while at the end of the laser pulse, $\rho_{1}$ is the contribution of ionization dissociation from $1 s \sigma_{g}$ to the total KER spectra. Thus $\rho^{*}$ in Fig. 2(a) is produced through highly excited vibrational states of $\mathrm{H}_{2}{ }^{+}$during ionization dissociation, namely, EID. It exhibits a kind of CE-KER pattern with equal-spacing fine structures.

The EID-KER spectra vary with the initial state and so do the relative values of the EID-KER spectra to the DCE-KER spectra. The DCE spectra depend on the survival probability of the initial state, while the EID spectra depend on the population probability of the excited and continuum states. Both rely on multiphoton excitation rate: the former is inversely proportional to it, while the latter is proportional to it. Since the multiphoton excitation rate from a lower vibrational state is much smaller than that from a higher vibrational state $[13,20]$, the relative values of the EID spectra to the DCE spectra increase rapidly with the initial vibrational state. This is more clearly illustrated in Fig. 3, where the total KER spectra $\rho$, the EID spectra $\rho^{*}$, and the DCE spectra $\rho_{1}^{11}$ are plotted for the $\mathrm{H}_{2}{ }^{+}$with a highly excited EV initial state $\left(n_{0}, v_{0}\right)=(1,11)$. The laser field parameters are the same as those in Fig. 2. It is shown that the EID spectra $\rho^{*}$ are now much larger than the DCE spectra $\rho_{1}^{11}$. The total KER spectra are mainly produced from EID. This is due to the large multiphoton excitation rate from the vibrational state $v_{0}=11$ to the highly excited and continuum states.

Finally, the EID spectra also depend strongly on the laser pulse. For a long-wavelength laser, the photon energy is 


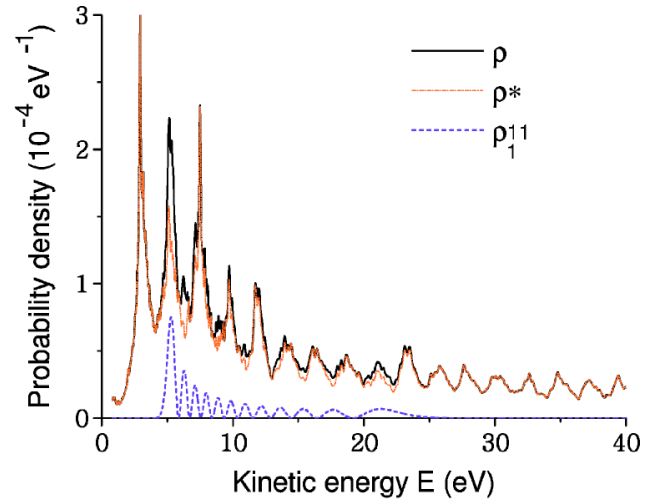

FIG. 3. Same as Fig. 2(a) but the initial state of $\mathrm{H}_{2}{ }^{+}$is a highly excited EV state $(1,11)$.

small, so is the peak separation in EID spectra. Because of the strong field effect, the peak width in the EID spectra may become broader with the increase of laser intensity. When the laser pulse duration decreases, the EID spectra may become smaller as a result of fewer populations on the excited and continuum states. Therefore, to observe the equalspacing fine structures in the EID-KER spectrum, it is re- quired that $\mathrm{H}_{2}^{+}$be prepared initially in a high vibrational state and that the laser be equipped with a shorter wavelength, intermediate intensity $\left(\sim 5 \times 10^{13} \mathrm{~W} / \mathrm{cm}^{2}\right)$, and a little bit longer pulse duration.

In summary, based on a high-precision 3D ab initio method, we have performed a detailed investigation of the process involving ionization dissociation in the fragmentation of $\mathrm{H}_{2}{ }^{+}$. A different kind of CE-KER spectrum, the EIDKER spectrum, is predicted, which consists of a sequence of peaks separated by one photon energy. The variations of the EID spectra with the initial state of $\mathrm{H}_{2}{ }^{+}$and the laser pulse are discussed. A scheme to observe the fine structures of EID spectrum is proposed. The EID phenomena studied in this paper is general and is expected to be valid for other molecules as well.

This work is partially supported by the Chemical Sciences, Geosciences, and Biosciences Division of the Office of Basic Energy Sciences, Office of Science, U. S. Department of Energy, and by the National Science Foundation. We acknowledge the Kansas Center for Advanced Scientific Computing for the support of supercomputer time.
[1] X. Urbain et al., Phys. Rev. Lett. 92, 163004 (2004).

[2] H. Niikura et al., Nature (London) 417, 917 (2002); Nature (London) 421, 826 (2003).

[3] H. Sakai et al., Phys. Rev. A 67, 063404 (2003).

[4] A. Staudte et al., Phys. Rev. A 65, 020703 (2002).

[5] H. Rottke et al., Phys. Rev. Lett. 89, 013001 (2002).

[6] L. J. Frasinski et al., Phys. Rev. Lett. 86, 2541 (2001).

[7] C. Trump et al., Phys. Rev. A 62, 063402 (2000); 60, 3924 (1999).

[8] K. Sndig, H. Figger, and T. Hnsch, Phys. Rev. Lett. 85, 4876 (2000).

[9] I. D. Williams et al., J. Phys. B 33, 2743 (2000).

[10] T. D. G. Walsh et al., Phys. Rev. A 58, 3922 (1998); J. Phys. B 31, 4853 (1998).

[11] A. Giusti-Suzor et al., Phys. Rev. Lett. 64, 515 (1990).

[12] P. H. Bucksbaum et al., Phys. Rev. Lett. 64, 1883 (1990).
[13] G. Yao and S.-I. Chu, Phys. Rev. A 48, 485 (1993).

[14] L. J. Frasinski et al., Phys. Rev. Lett. 83, 3625 (1999).

[15] T. Seideman, M. Y. Ivanov, and P. B. Corkum, Phys. Rev. Lett. 75, 2819 (1995).

[16] X. M. Tong, Z. X. Zhao, and C. D. Lin, Phys. Rev. Lett. 91, 233203 (2003); Phys. Rev. A 68, 043412 (2003).

[17] S. Chelkowski, P. B. Corkum, and A. D. Bandrauk, Phys. Rev. Lett. 82, 3416 (1999).

[18] M. Tarisien et al., J. Phys. B 33, L11 (2000).

[19] F. A. Rajgara et al., J. Phys. B 37, 1699 (2004).

[20] Z. Zhou and S.-I. Chu, Phys. Rev. A (unpublished).

[21] X. Chu and S.-I. Chu, Phys. Rev. A 63, 013414 (2001).

[22] S.-I. Chu, Chem. Phys. Lett. 167, 155 (1990).

[23] T. F. Jiang and S.-I. Chu, Chem. Phys. Lett. 240, 278 (1995).

[24] M. R. Hermann and J. A. Fleck, Jr., Phys. Rev. A 38, 6000 (1988). 